\title{
UN INFORME DE PEDRO DE RIBERA PARA NUEVA ESPAÑA: EL ABASTECIMIENTO DE AGUA A GUADALAJARA
}

\author{
Álvaro Recio Mir \\ Universidad de Sevilla y Autónoma de Chile
}

\begin{abstract}
El proyecto que el arquitecto franciscano Pedro Antonio de Buzeta planteó para abastecer de agua a la ciudad novohispana de Guadalajara fue enviado a Madrid para que lo supervisase el consejo de Indias. De sus aspectos técnicos se ocupó el arquitecto madrileño Pedro de Ribera, que firmó una interesante crítica a la empresa en 1735. Entre otras cuestiones, hizo una defensa de los acueductos subterráneos, demostró su erudición y planteó diversas correcciones al proyecto de Buzeta.
\end{abstract}

Palabras clave: Pedro de Ribera; Fray Pedro Antonio de Buzeta; Acueducto; Guadalajara; Nueva España.

\section{A REPORT BY PEDRO DE RIBERA FOR NEW SPAIN: WATER SUPPLY TO GUADALAJARA}

The project proposed by the Franciscan architect Pedro Antonio de Buzeta to supply water to the New Spanish city of Guadalajara was sent to Madrid for review and supervision by the Council of the Indies. The Madrid architect Pedro de Ribera dealt with its technical aspects, and in 1735 he wrote an interesting critique of the plan. Among other questions, he defended the use of underground aqueducts, demonstrated his erudition and considered diverse improvements to Buzeta's project.

Key words: Pedro de Ribera; Monk Pedro Antonio de Buzeta; Aqueduct; Guadalajara; New Spain.

"Cuando se fundó esta ciudad en la orilla derecha del riachuelo que nace al Sur se creyó que sus aguas serían suficientes para abastecer la población”. Con estas palabras da comienzo la Historia de la introducción de agua en Guadalajara, publicada en 1842 y que se atribuye a Manuel López Cotilla. Pronto se vio que ese cauce era insuficiente para la capital tapatía, por lo que se inició la empresa de su abastecimiento, que se dilató a lo largo de gran parte del período virreinal. La documentación que narra esta empresa hace referencia, desde la fundación de la ciudad, a los numerosos y frustrados intentos por encontrar agua, habituales por cierto en otras ciudades tanto de España como de América y que cabría relacionar con los problemas de salubridad del Antiguo Régimen. En cualquier caso, en un principio, en Guadalajara se destinó a su abasto el manantial llamado Agua Blanca, a un cuarto de legua al sur de la población, donde hoy se encuentra la plaza de la Bandera. No obstante, ya en 1563 la audiencia solicitó un informe 
sobre la obra que se habría de ejecutar para llevar el agua hasta el centro de la ciudad, que fue el primer intento conocido de hacer un acueducto y también el primer fracaso al respecto ${ }^{1}$.

En 1597 una real cédula de Felipe II otorgó una merced de tierras para empezar las obras, lo que parece que se produjo de inmediato. Incluso, se cree que ese año una atarjea de cal y canto llevó el agua desde los manantiales de Los Colomos al convento de Santo Domingo, de lo cual han quedado vestigios arqueológicos. Este primer acueducto debió de funcionar poco tiempo, seguramente por su deficiente factura o por reducirse el nivel de su acuífero. En cualquier caso, no volvió a retomarse la preocupación por el abastecimiento de agua hasta 1640, cuando la ciudad mostró a Felipe IV su desconsuelo al respecto. Ante ello una nueva real cédula dispuso que "se repartiera su costo entre los vecinos", pero nada se hizo, de manera que tuvieron que pasar otras tres décadas para que el asunto se reactivase. Así, en 1672 Francisco Calderón Romero, presidente de la audiencia de Guadalajara, por petición de su fiscal y con acuerdo del obispo, propuso al rey que el comercio del vino de coco y del mezcal en la ciudad fuese estancado y arrendado, aplicando su producto a pagar la conducción de agua, de la que decía que se había iniciado "a su costa". De nuevo, esta propuesta resultó un fracaso².

Años más tarde "un abogado de Zacatecas llamado don Alonso de León, muy preciado de ingenio, ofreció en el año 1692 meter el agua en esta ciudad traiéndola de unos manantiales que entonces avía al sur de esta corte”. En esta ocasión sí se llegó a hacer la obra, pero con muy escaso resultado. Tuvieron que sucederse otros intentos fallidos hasta que en 1726 el presidente de la audiencia, Nicolás de Rivera, mostró al rey "los grandes inconvenientes de la falta de agua", rogándole que se ampliasen veinticinco años las prerrogativas sobre el vino de coco y el mezcal. El monarca emitió una nueva real cédula en 1727 en respuesta a tal petición, instando a la reanudación de la empresa. Por su parte, la audiencia dictó un auto en 1729 por el que mandaba ejecutar la referida real cédula, de manera que nombró como director de la obra a su oidor, el licenciado don Juan Rodríguez de Albuerne, marqués de Altamira, que fue quien puso en marcha la esperada empresa hidráulica ${ }^{3}$.

Albuerne encargó la obra al arquitecto fray Pedro Antonio de Buzeta, que se encontraba en Nueva España pidiendo limosna para concluir su convento de San Francisco, que él había levantado en la localidad española de Sanlúcar de Barrameda. Como especialista que era en conducciones de aguas, entre otras obras, había llevado a cabo en el virreinato novohispano el abastecimiento a Veracruz, lo que le había dado cierta fama ${ }^{4}$.

Tras recorrer los alrededores de Guadalajara, Buzeta proyectó un acueducto con dos ramales subterráneos. El primero, orientado a poniente, sería una mina o galería de ochocientas varas, ejecutada a partir de pozos abiertos cada cincuenta varas, que luego serían unidos subterráneamente. A ello se sumaría un segundo ramal, de orientación Sur-Norte, de más de mil varas y "con cuia línea traviesa se cortarán muchos veneros derramados y dispersos por el llano y algunos o parte de los manantiales arriba expresados, que todos han de venir a juntarse en la otra línea que baja del poniente a esta ciudad". Ambos ramales confluirían en una caja de agua en las inmediaciones de la ciudad, a partir de la cual se repartiría el preciado líquido mediante fuentes públicas y privadas por Guadalajara ${ }^{5}$.

\footnotetext{
${ }^{1}$ López Cotilla, 1842: 3. Existen ediciones recientes de esta obra como López Cotilla, 2013. Sobre el abastecimiento de agua a Guadalajara véase también JALOMO AGUIRRE, 2011: 154 y ss.

${ }^{2}$ López Cotilla, 1842: 3 y 4 y Jalomo Aguirre, 2011: 154.

3 López Cotilla, 1842: 4-6 y Jalomo Aguirre, 2011: 155 y 156.

${ }^{4}$ Sobre Buzeta, además de la bibliografía citada hasta ahora, remitimos a Ramos, 1942; VelázQuez-GazTelu, 1995: 158 y ss. y LAORDEN RAMOS, 2008: vol. I, 36 y ss.

${ }^{5}$ López Cotilla, 1842:7 y Jalomo Aguirre, 2011: 156 y 157.
} 
De este proyecto conservamos un rasguño, que se fecha, igual que el proyecto, en 1732. Se trata de un sumario borrador, croquis o apunte de lo observado en el entorno de Guadalajara por Buzeta y de su plan para llevar el agua al centro de la misma. Su carácter provisional lo evidencian los tachones que vemos en él, que sus inscripciones estén escritas en distintos sentidos y que haya diferentes tipos de letras y tintas. En cualquier caso, todo parece indicar que los "Ojos del agua del río Izapopán" a los que se alude eran el ramal de poniente del acueducto, mientras el tanque que aparece al Sur, estaría en relación con el ramal Sur-Norte. Ambos ramales se unirían a poniente, para entrar en la ciudad por el convento de Jesús María hasta llegar a la plaza mayor ${ }^{6}$ (fig. 1).

Cabe preguntarse por qué este esbozo formó parte del expediente enviado a España para ser juzgado en Madrid. Sin duda, Buzeta tenía mayores cualidades gráficas que las mostradas en este caso, como prueba otro dibujo suyo de 1741 de la obra terminada y que con posterioridad también fue enviado a España ${ }^{7}$. Además, como veremos más adelante, el expediente que ahora analizamos contó con otro plano que no se conserva. En cualquier caso, este rasguño es la primera planta de la ciudad de Guadalajara, debiendo resaltarse que fue obra de Pedro Antonio de Buzeta $^{8}$.

La construcción del acueducto se prolongó una década, concluyéndose en 1741. El ritmo de trabajo fue intenso, de manera que ya en 1733 se vieron los primeros resultados. No obstante, Buzeta recibió muchas críticas que despreció con llamativa soberbia. Así, a los que cuestionaban su labor los llamó "medidores de tierras mui preciados de geómetras, otros mui conceptuados de filósofos". De otros dijo que le injuriaban por declarar que "estaba engordando la mula para escaparme", cuando en realidad estaba "enfermo y baldado de pies y manos por avérseme derretido las reumas con la fuerza de los soles". También se dijo que "avía enfermado de la pesadumbre de aver conocido el disparate de la empresa". Buzeta respondió a todo ello afirmando que "llegando a mi noticia de semejantes oprobios e injurias, sin respeto al santo hábito que visto, me violentaron tanto que sin poder vuestra merced contenerme hize meterme en un forlón y vine a poner la primera piedra, para que se desengañasen de que no avía enfermado por desconfianza alguna de la obra"9.

No obstante, la crítica más interesante al proyecto de Buzeta para abastecer de agua a la ciudad de Guadalajara fue la que planteó el arquitecto madrileño Pedro de Ribera. Su origen se encuentra en que el proyecto del franciscano fue enviado a Madrid, para que lo supervisase el consejo de Indias, el cual lo remitió a Ribera para que informase del mismo ${ }^{10}$.

En su informe empieza señalando Ribera que recibió el expediente mandado por Albuerne y que contenía "la carta y mapa formado por el padre fray Pedro Antonio de Buzeta, del orden de San Francisco, que parece ser el maestro arquitecto que ha principiado la obra". Describe luego el arquitecto madrileño la labor de Buzeta: "tiene abiertos los dos ramales que miran del sur al norte para cortar las venas por donde descienden de los montes al llano las aguas que en

\footnotetext{
${ }^{6}$ Archivo General de Indias (A.G.I.), Mapas y planos, México, 127. Véanse también CALDERÓN QuiJanO, 1984: 62-64.

${ }^{7}$ Sobre tal dibujo, que sólo mencionamos al ser posterior al proyecto de Buzeta y al informe de Ribera, véase CALDERÓN QUiJANO, 1984: 64-66.

${ }^{8}$ No recoge tal autoría ni CALderón Quijano, 1984: 62-64, ni su ficha en A.G.I. Mapas y planos, México, 127.

9 A.G.I, Guadalajara 420, fols. 250 y 250 vto.

10 Este informe fue sólo mencionado por Angulo, lo cual no ha sido recogido por la bibliografía que se ha ocupado de Ribera y que aludiremos más adelante. Véase ANGUlo ÍñIgueZ, 1939: vol. 1. ${ }^{\circ}, 341$. Por nuestra parte, recogemos el informe prácticamente completo o, mejor dicho, la copia que del mismo se conserva en el Archivo General de Indias, salvo alguna reiteración y fórmula diplomática que nada aportan a su contenido. Por la significación y expresividad del texto del informe hemos creído más oportuno analizarlo a medida que lo transcribimos en el cuerpo del artículo, que reproducirlo en un apéndice documental al final del mismo.
}

Arch. esp. arte, LXXXVII, 348, OCTUBRE-DICIEMBRE 2014, 351-364 ISSN: 0004-0428, eISSN: 1988-8511, doi: 10.3989/aearte.2014.22 


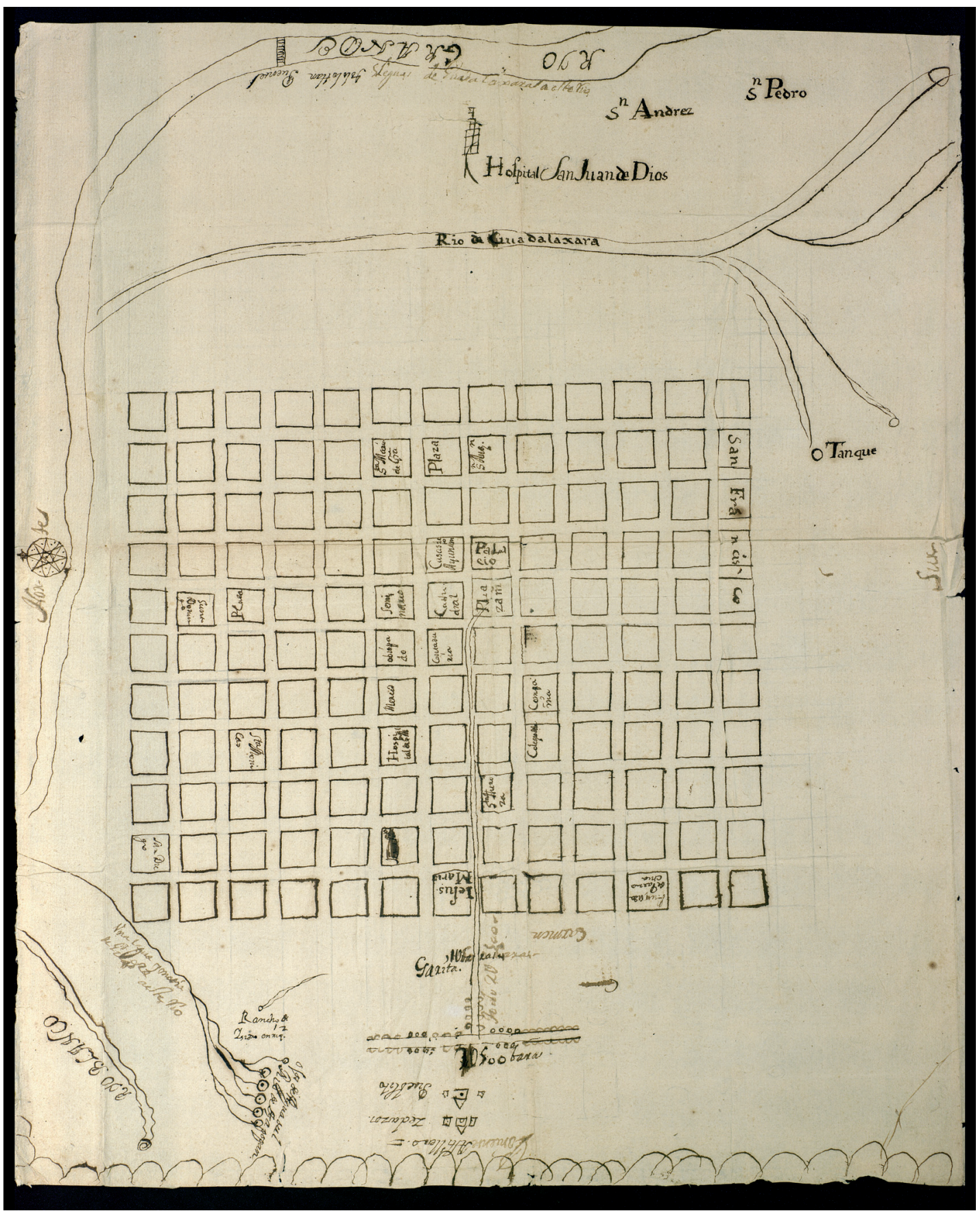

Fig. 1. Fray Pedro Antonio de Buzeta, Proyecto para el abastecimiento de agua a Guadalajara, Nueva España, 1732, Archivo General de Indias, Mapas y planos, México, 127. 
ellos nacen subterráneas, pues aunque no se reconocen salir como fuentes en sus faldas, es lo natural que por las costras o venas de los géneros de la tierra o piedra se van sumergiendo y buscando lo más profundo de los valles" 11.

El acueducto de Buzeta le sirvió a Ribera para hacer una digresión sobre las conducciones de agua subterráneas, de las que era firme defensor al estar avaladas por la experiencia. En concreto, dice que ello "lo experimentó León Bauptista Alberto y lo explica en el libro décimo, capitulo 4, en que dice que los indicios de hallar el agua es que los sitios sean cóncavos como un vaso, que siempre está prompto a recibir y no como en los altos, que a estos los hiere el sol y con más fuerza los deseca y consume el humor radical y si hallasen algunas serán crudas, viciosas y saladas, lo que no subcederá en las faldas de los montes que miran al norte si se cavase o hiciesen pozos, se hallará el agua con facilidad y mui segura también". A ello añadía que "los montes que se cubren de nieve dan más abundante el agua y mucho más si en sus alturas tienen llanos y muchos prados, son mui abundantes de aguas y por lo común de semejantes montes nazen los ríos. También he advertido que las fuentes más copiosas sólo nazen a la redondez de los montes, que sus suelos son de terreno firme y en lo alto tengan prados" 12 .

Sumaba Ribera otra justificación histórica, recordando que Tácito narró la peregrinación de Moisés "por desiertos y acosado por la sed, por congetura de las hierbas halló las venas de las aguas". Aún añadía el franciscano un tercer ejemplo histórico: "Emilio como tenía el egército junto a Olipapo y tenia falta de agua la alló amonestado por la verdura de las selvas, las más verdaderas señales y más experimentadas son las partes donde se crían el salce, cañas, juncos y las hiedras y todas aquellas que necesitan de grande abundancia de humedad para su producción" 13 .

Todo lo anterior llevaba a Ribera a valorar positivamente "la elezión de abrir pozos en valle o llano que ha empezado a ejecutar nuestro padre entre dos cerros o faldas de los dos montes que parece están entre el sur y norte son mui bien prevenidas las cortaduras con las cuales pueden sangrar todas las venas de la tierra por donde descienden las aguas al llano, donde fabrica los pozos y minas" 14 .

Para entender esta defensa de los acueductos subterráneos hay que señalar que entonces había una oposición mayoritaria en España y en Nueva España a tal forma de abastecimiento de agua, prefiriéndose los tradicionales acueductos sobre arcos, de los que tan espléndidos ejemplos había a ambos lados del Atlántico, habiendo alcanzado el rango de mitos los de Segovia y Zempoala ${ }^{15}$. De igual modo, hay que tener en cuenta, que esa era la forma habitual de conseguir el abasto de agua a Madrid, como veremos más adelante.

En cuanto a Alberti, alude Ribera a su De re aedificatoria, cuyo capítulo cuarto del libro décimo trata, en efecto, de los "indicios de hallar la agua escondida y dos maneras de cavalla" 16. En cuanto a la cita de Tácito, refiere el episodio en el que Moisés, mientras guiaba a Israel por el desierto, encontró agua para su pueblo que estaba sediento, episodio narrado por el citado autor latino en el libro quinto de sus Historias ${ }^{17}$. La tercera alusión histórica de Ribera es a la batalla de Pidna, que puso fin a la Tercera Guerra Macedónica. En ella el ejército romano se encontraba en el 168 a. de Xto. en Elpeo y ante la escasez de agua su líder, el cónsul Lucio

\footnotetext{
11 A.G.I., Guadalajara 420, fols. 257 y 257 vto.

12 A.G.I., Guadalajara 420, fols. 257 vto. y 258.

13 A.G.I., Guadalajara 420, fol. 258.

14 Ibidem.

15 Sobre esta oposición véase Varios autores, 1994, vol. 2. ${ }^{\circ}, 164$.

${ }^{16}$ Citamos por la traducción de Francisco Lozano, que pudo usar Ribera, AlBERTI, 1582: 310 y ss.

17 Véase TÁcito, 1744: vol. 3. ${ }^{\circ}, 450-460$.
} 
Emilio Paulo, mandó cavar unos pozos que solucionaron el problema, lo que a la postre permitió la victoria romana sobre los macedonios ${ }^{18}$.

No obstante, a pesar de su apoyo a los acueductos subterráneos, Ribera supeditaba su aprobación al proyecto de Buzeta a "que demuestre" que lo que "en su plan tiene la letra D, donde dize tiene recogidas las aguas de los dos ramales, tendrá salida para que no se detengan sino que corran sin cesar" 19 . Ello prueba que Buzeta no sólo mandó a Madrid el rasguño que antes aludimos, ya que en él no hay ninguna letra D. Cabe suponer que la misma haría alusión, en un proyecto perfectamente dibujado, al arca de agua, de la que lógicamente advierte Ribera que debía tener continuidad con el resto del acueducto para que el agua llegase a término y no se detuviese en ese punto.

Otra objeción que planteó Ribera a Buzeta fue su precipitación en la medición del agua, de lo cual dijo que "la cantidad de agua que da lo minado y si aumenta o disminuye" era cuestión de tiempo, "pues los tres meses que dize ha estado corriendo no es tiempo suficiente para una experiencia de tanta importancia y tan grandes intereses, pues la experiencia nos ha hecho conocer la incertidumbre de semejantes obras, pues en nuestros tiempos hemos experimentado que los veneros o nacimientos de aguas de muchos años han faltado en un todo, por la razón de haber bajado las aguas en dichos nacimientos, ya por lo ardiente del sol, ya porque las costras de las venas han tomado otro curso las aguas, por haber hallado otra parte que con más facilidad ha buscado su natural el grave de su peso y asi se sabe que en muchas partes han faltado por el invierno de prompto las aguas de sus fuentes y otras que de prompto en el estío también han faltado y otras que después de muchos años que se secaron han vuelto aparecer con más abundancia" 20 .

Las palabras de Ribera fueron premonitorias, ya que el acueducto de Buzeta, que culminó con éxito, pronto resultó inoperante. Apenas terminado ya requirió de obras que se prolongaron todo el siglo XVIII y hasta después de la independencia de México. Ni siquiera así se solucionaron los problemas de abastecimiento de agua a Guadalajara, por lo que a principios del siglo XX se levantó un nuevo acueducto durante la presidencia de Porfirio Díaz ${ }^{21}$.

También critica Ribera la altivez de Buzeta por llamar a "todos los arquitectos que ponen en evidencia las dificultades en materia de las aguas que están escondidas... temerarios en sus dictámenes". Ribera explica que "proponer las dificultades que a cada uno se le ofrecen no es motivo para vituperarlos con las voces de fanáticos, incrédulos, desafectos, cosas que en los seglares pareciera demasiada temeridad, quanto más parecerá en un religioso que viste el hábito de nuestro padre San Francisco y aún extraño más que diga que sólo su dictamen es el que se tiene que aprobar según las experiencias y observaciones que hizo dicho padre y los pozos que egecutó para su reconocimiento"22.

Ribera también dudaba del cálculo de Buzeta sobre la cantidad de agua que correría por las galerías que había construido. Decía en concreto: "no ignora dicho padre que las aguas de dichos pozos, aunque estén con abundancia, no teniendo desaguadero por donde tome su curso el agua que se halla oprimida en el centro de las venas y como ésta desea salir sin opresión inmediatamente que se le abra mina por ella toma su curso como quien desea la libertad y así luego da abundancia toda la que tienen sus cóncavas hasta que da la que desciende de los montes o faldas de ellos y ésta no es mui segura hasta que aia pasado un año o dos con que no sé por donde el

\footnotetext{
18 No hace referencia en este caso Ribera a fuente alguna sobre este episodio histórico.

19 A.G.I., Guadalajara 420, fols. 258 y 258 vto.

20 A.G.I., Guadalajara 420, fols. 258 vto. y 259.

21 Véanse López Cotilla, 1842: 7 y ss. y Jalomo Aguirre, 2011: 160-165. También remitimos a Romo, 1888, 34 y 35; Romero de Terreros, 1949: 127-129 y Varios Autores, 1994: vol. 2. ${ }^{\circ}, 165$.

22 A.G.I., Guadalajara 420, fol. 259.
} 
dicho padre con término tan corto como el de tres meses quiera asegurar serán seguros y estables quando todos los hombres prácticos y theóricos no lo aseguran, porque de lo oculto hasta ahora no ha habido quien lo asegure con tantas veras como el padre lo asegura y así los dictámenes de los más ínfimos no se deben despreciar quanto ni más los de hombres científicos como lo serán todos los que han dado sus dictámenes en esa obra"23.

Vuelve a fundamentar su postura al respecto Ribera en la historia al señalar: "tenemos tantos y tan grandes ejemplares en lo antiguo y moderno que lo que muchos hombres insignes en cosas fáciles se han hallado perdidos y un mero práctico y aún sin ninguna inteligencia ni conocimiento han dado en la dificultad y valga por todos los subcedidos el que le pasó a un hombre tan grande como lo fue Domenico Fontana, uno de los maiores arquitectos que tuvo Roma como demuestran sus libros y especialmente el que hizo sobre las máquinas que egecutó para mudar al abuja que en aquellos tiempos estaba detrás de la iglesia de San Pedro y la pasó a la gran plaza que está en la fachada de dicha iglesia, para cuio efecto concurrieron todos los maiores ingenios que en aquellos tiempos florecieron y por fin se egecutó como dicho Domenico Fontana propuso y habiendo tomado sus medidas y alturas para enderezarla se halló al tiempo que se hallaba presente toda la corte romana el que no la podía enderezar porque no tubo presente lo que habian de dar de si las cuerdas que pendían de las arganas o garruchas para su elevación por cuio motivo quiso precipitarse y arrojarse de los andamios abajo por parecerle que padezía su honra a vista del senado tan grande y viéndole tan triste y que quería arrojarse, uno de aquellos peones que el ayudaban le preguntó la causa y él se la dijo y entonces el tal le respondió que si eso le daba cuidado que estaba remediado mui breve, volvió sobre sí el artífice y le preguntó de qué suerte lo havía de remediar y le respondió el tal operario que subiesen unos cubos de agua y con efecto los subieron y el tal empezó a regar las cuerdas y con la humedad volvieron a encoger dichas cuerdas lo que habian dado de sí, con lo cual enderezó su abuja y quedó con la honra que fue tan pública que le premiaron como merecía obra de tanta grandeza, también se dice que el pago que dio al operario fue echarle de la torre abajo porque no digese havía sacádole de tanto aprieto y que no hubiese quien adelantase a su discurso pago que en todo tiempo da el mundo por lo que digo que no se debe tener en que sea vituperio el que cada uno de su dictamen pues el más preciado de que sabe ignora más" 24 .

Tras tal comparación y, particularmente, tras la moraleja final, Ribera planteaba otras dudas técnicas sobre el proyecto de Buzeta, a la vez que, de nuevo, criticaba su soberbia. Así, decía en primer lugar que en su carta "tiene nivelado desde lo profundo de los pozos que tiene egecutados $y$ arcas donde se juntan los dos ramales que demuestra el plan señalado con la letra $F$, aunque no dice qué cantidad de agua sale al presente ni qué desnivel tiene y qué altura tendrán en la ciudad los surtidores de las fuentes y si desde el estanque grande señalado con la letra B donde parece ha de repartir las tres minas o cañerías que han de llevar el agua a la ciudad y casa del señor arzobispo comunidades y otros particulares". Es decir, omitía información esencial para la emisión de un informe, ya que no revelaba la cantidad de agua proporcionada por el acueducto ni el desnivel que habría de tener para que corriera. Ello le lleva a Ribera a dudar de Buzeta y a criticar su soberbia, "pues quien sabe nivelar con nivel graduado, báculo mensorio, quadrante geométrico y nivel de agua y tan general en todos géneros de fabrica de albañilería, mampostería y cantería no deja que dude el arquitecto consumado aunque siempre he oydo decir que la alabanza propia quita mucha parte de estimación al que la pronuncia vituperando a los demás que proponen las dificultades con términos mui indignos y lo que me admira más es que dicho religioso que profesa la humildad no la practique con el próximo pues da a entender que solo él sabe lo que son semejantes obras, pues dice contra la obra de la conducción de Nuestra Señora

23 A.G.I., Guadalajara 420, fols. 259 y 259 vto.

Arch. esp. arte, LXXXVII, 348, OCTUBRE-DICIEMBRE 2014, 351-364 ISSN: 0004-0428, eISSN: 1988-8511, doi: 10.3989/aearte.2014.22 
de los Remedios de Méjico se quedó sin agua por no seguir su dictamen se perdió la obra como también en la conduzión del agua de Nuestra Señora de Guadalupe del mismo Méjico que se quedó sin ella y sobre todo tira al abogado que yntentó traer el agua a esta ciudad en los años de 1692, como también con el maestro que el año de 1700 ofreció entrar el agua en dicha ciudad con el término de que era la misma con que el abogado havía engañado a dicha ciudad faltando en todo a la charidad de poner en público los defectos de los demás y, por último, no está seguro aún el religioso que en los años pasados de 1733 trujo la audiencia para reconocer si se podría traer agua a dicha ciudad y dando su dictamen sin meterse en otra cosa, motivos que me hacen dudar del azierto de la obra, pues siempre se ha visto que quien más sabe más dificultades se le ofrecen en todas especies de artes lo que no le subcede al ignorante que éste como no conoce las dificultades no se le ofrecen reparos y le parece que todo lo puede conseguir y que las que los demás proponen son por odio y mala voluntad al que ejecuta la obra"25.

Ribera culminaba su informe diciendo que "supuesto lo referido y para exponer mi dictamen esta materia como quiera que puesta en planta y principiada la dicha fábrica toda la duda o dificultad que pueda ofrecerse consiste en la permanente consistencia o duración de las aguas, respecto de que al tiempo que escribió la carta el padre arquitecto corrían poco tiempo habían y tal que podía recelarse su extinción este ynconbeniente considera le habrá superado el transcurso de más de dos años que serán tres quando llegue a Guadalaxara la resolución con que ya se habrá verificado el uno u otro extremo, esto es o el de la duración de las aguas por ser naturales y peregnes o el de su falta por ser accidentales o superficiales, le parece que la experiencia habrá apartado toda duda y que para el primer caso se podrán dar todas las providencias necesarias para la prosecución y fenecimiento de la obra, las que no servirán en el segundo caso pues se verificará ser ynútil" 26.

El transcurso del tiempo, por tanto, habría de aclarar la viabilidad del proyecto y otra duda que planteaba Ribera: "no excusa hacer presente que resultando por el mapa o plan haverse hecho las aberturas en el cerro o monte, en cuia superior situación se dice haver algunos pueblos de indios, los que puede ser mui regular padezcan el perjuicio de que llamando las aguas a la parte inferior con las aberturas egecutadas carezcan de las que tenían para su manutención lo que asimismo habrá verificado la experiencia en el transcurso del tiempo, lo que deberá tener presente el arquitecto para sino hubiesen otros sitios donde surtirse de agua dichos pueblos ver la providencia que pueda ofrecer el terreno para quitar este inconveniente"27.

Además del abastecimiento de agua a dichos pueblos de Indios, que la obra de Buzeta podía poner en peligro, Ribera añadía una última advertencia: "también se debe tener presente que el plantío de árboles que dice ha empezado a poner para la diversión y paseo se desvie de las zanjas, minas, cañerías que se hiciesen pues estos son perjudiciales a semejantes obras pues sus raices se extienden y bajan a buscar la humedad del agua que pasa por ellas y se mazizan de raices así las minas como las cañerías como se experimenta en esta villa en todas las que pasen por junto a donde ai árboles que se suelen sacar rayces que tienen 16 o 18 varas de largo y gruesas quanto caben en la cañería de suerte que las troncha a cada paso". Con ello terminaba su informe Pedro de Ribera, que lo firmaba en Madrid, el 13 de agosto de $1735^{28}$.

La pretensión del informe enviado por Albuerne desde Guadalajara a Madrid era conseguir tanto financiación como respaldo del consejo de Indias para acabar la obra ante las dudas que había suscitado. Albuerne, en concreto, proponía al consejo "destinar arbitrios a efecto de juntar

25 A.G.I., Guadalajara 420, fols. 261 y 262. Sobre los referidos acueductos de Guadalupe y de los Remedios, referidos en el informe de Ribera, remitimos respectivamente a RUIZ GOMAR, 1983 y a ROMERO DE TERREROS, 1949: 83-91.

26 A.G.I., Guadalajara 420, fols. 262 y 262 vto.

27 A.G.I., Guadalajara 420, fols. 262 vto. y 263.

28 A.G.I., Guadalajara 420, fol. 263.

Arch. esp. arte, LXXXVII, 348, OCTUBRE-DICIEMBRE 2014, 351-364 ISSN: 0004-0428, eISSN: 1988-8511, doi: 10.3989/aearte.2014.22 
los fondos necesarios, sin costa de la real hazienda ni perjuicio del terreno". Mientras tanto, Buzeta en el mismo expediente declaraba que la obra había sido tildada de "imposible y disparatada", a pesar que "hacía tres meses que corría bastante agua sólo con haber minado 160 varas y que costándose y trabajando hasta 1400, que por entonces se pensaba, abría bastante para las fuentes públicas, 14 comunidades, real palacio, cárzeles, casa del señor obispo y las demás de los vecinos particulares que quisiesen comprar alguna merced de agua" 29 . Por tales expectativas y por las referidas críticas era por lo que se solicitaba el refrendo técnico, institucional y financiero a la empresa constructiva.

El consejo de Indias señaló que, una vez comprobada "la duración de las aguas por ser naturales y peregnes, se podrán dar todas las providencias necesarias para la prosecución y fenecimiento de la obra", las cuales lógicamente no se darían "si por ser accidentales y superficiales faltasen". También se afirmaba que habría de plantearse la posibilidad, como había advertido Ribera, de que algunos de los referidos pueblos de indios "padezcan el perjuicio de que llamando las aguas a la parte inferior carezcan de las que toman". De igual modo, el consejo recordaba, siguiendo de nuevo el informe de Ribera, que "el plantío de los árboles que se supone haber empezado para la diversión del paseo se desvie de las sanjas y cañerías que se hiziesen por ser perjudiciales, pues sus raizes se extienden y bajan a buscar la humedad del agua que pasa por ellas y se macizan de raizes asi las minas como cañerías". Concluía advirtiendo que sólo con estas "prevenciones... se deberá pasar a la finalización de esta obra y para este fin valerse de los referidos arbitrios" $" 30$.

Junto a tales pareceres, el consejo de Indias mandó a la audiencia de México que "evaque en todas instancias el pleito pendiente ante el juez general de bienes de difuntos de aquel distrito sobre la cobranza de los 13 mil y tantos pesos pertenecientes a la real hazienda en los bienes del presidente don Thomás Therán". A ello sumaba la orden de que, una vez cobrados, "se les remitan al oydor Albuerne para que continúe los trabajos de esta obra de que está encargado y el mismo encargo se hará al fiscal de la audiencia de México, otra al presidente y oficiales de Guadalaxara, para que continúen en aplicar a esta fábrica los arbitrios que se conzedieron los años de 723 y 731 del prometido de las carnes y lizencias de saca de ganados y que se prorroga por otros doze años cumplidos que sean los concedidos". Todos estos fondos habrían de ponerse "a disposición del oydor Albuerne, quien debe proceder en el manejo de ellos y dirección de toda esta obra con absoluta inhivición de los tribunales y demás arbitrios" 31 .

Otra orden era remitida "al presidente de la audiencia y ciudad de Guadalaxara, en que refiriendo lo mandado el año de 1640 se les diga haver llegado el caso de que se ponga en práctica el repartimiento entre los vecinos de los 16 mil pesos entonces considerados por precisos para esta obra y que en el prozedan con la justificación y proporzión prevenida por leyes". Se enviaría otra orden a Albuerne "avisándole el recibo de todas sus representaciones, dándole gracias por su zelo y las órdenes que queda expresado se han de dar para que lo tenga entendido como también los reparos y advertencias hechas por don Pedro de Rivera a fin que las tenga muy presentes y que por lo que mira a la prórroga del estanco de vinos se queda considerando la providencia que se hubiere de aplicar y se le prevendrá que hecho cómputo prudenzial de lo que importaran los arbitrios destinados y que ahora se aplican para esta obra y de lo que se fuere adelantando en ella dé cuenta de la que faltara para su conclusión". También se ordenó "al virrey que los 13 mil y tantos pesos y demás caudales aplicados a esta obra no los divierta en otra cosa". Y, por último, se indicó que "se hará consulta a su majestad para que se

\footnotetext{
29 A.G.I., Guadalajara 420, fols. 264 y 264 vto.

30 A.G.I., Guadalajara 420, fols. 264 vto. y 265.

31 A.G.I., Guadalajara 420, fol. 266.
}

Arch. esp. arte, LXXXVII, 348, OCTUBRE-DICIEMBRE 2014, 351-364 ISSN: 0004-0428, eISSN: 1988-8511, doi: 10.3989/aearte.2014.22 
digne prorrogar por otros doze años el arbitrio del vino de cocos para esta obra expresando la utilidad que se seguirá de ella"32.

Además de tal soporte financiero, en el expediente que analizamos, se encuentra la contestación de Albuerne a los informes solicitados por el consejo de Indias sobre las gestiones realizadas para cobrar la deuda del pleito de Tomás Terán. A ello añadía que quedaba "advertido de los bien premeditados reparos y justas prevenciones que en la real cédula a mi dirigida se hacen por lo tocante a la obra y en la angustia del tiempo sólo puedo decir que a más de tres años esta corriente el desagüe de los pozos y permanente el agua bastante para tres fuentes que se logrará con maior abundancia dilatando más las minas sin perjuicio alguno de los pueblos que están en la parte superior ni del plantío de árboles que se hizo a uno y otro lado de dicha mina en bastante distancia de ellas sin que puedan perjudicarla"33.

De igual forma, Albuerne, además de señalar la permanencia del agua y de que se habían seguido los postulados de Ribera, informaba sobre la cuestión económica, de la que decía que, a pesar de que "fray Pedro Buzeta, religioso lego del orden de San Francisco, maestro de la obra se vio necesitado de pasar a México donde se halla, por lo que no puedo hacer cómputo prudencial de lo que será necesario para la perfección de la obra", afirmaba que "si los efectos aplicados fueran promptos abría lo suficiente, pero los 13.260 pesos del presidente Therán no se han cobrado ni ay certeza de que se cobren los 18.000 pesos prorrogados en los doze años del prometido del abasto de carnes de esta ciudad necesitan de 17 años para devengarse por faltar de cumplirse cinco de los doze primeramente concedidos, los 16.000 mandados repartir entre vecinos no podrán ser en la mayor parte exigibles y el procedido de las licencias para extracción de ganados por las grandes mortandades que ha habido de estos ha bajado tanto que apenas sube de 200 pesos al año que es la única cantidad más prompta con la de los 1.500 pesos en cada un año del prometido de carnes pero ambas no pueden ser suficientes para la continuación de la obra" 34 .

Por último, además de los referidos apuros financieros, Albuerne informaba del estado de la obra: "hállase ésta en estado de hazer las cañerías para el repartimiento del agua conduzida en la tarjea a las fuentes con más de 4.000 caños prevenidos a este fin y suspendido su logro por falta de dinero prompto como sería el procedido del estanco de vinos de cosos y mescal". Por ello pedía que se pagase "desde el tiempo que cesó este arbitrio y por el tiempo y con las calidades que en mi consulta de 4 de enero de 1732 tengo suplicado a vuestra majestad". Aún advertía antes de terminar que: "en el tornaviaje de flota avisaré lo que ocurriere con más individualidad y justificación de todo que ahora no me permite la estrechez del tiempo que llevo referido y sobre todo mandará vuestra majestad lo que fuere más de su real dignación”. Se despedía deseando que "Dios guarde la cathólica y real persona de vuestra majestad como la Christiandad ha menester" y lo firmaba Juan Rodríguez de Albuerne, el 8 de mayo de 1736, en Guadalajara, Nueva España ${ }^{35}$.

Un análisis de conjunto del informe de Pedro de Ribera sobre el abastecimiento de agua a Guadalajara debe empezar señalando que la elección del arquitecto tiene significación en sí misma. Sin duda, la empresa a valorar era de gran envergadura y se había frustrado desde el siglo XVI, por lo que era necesario un arquitecto de renombre que no sólo la aprobara, si no que le diera su impulso definitivo. En este sentido, cuando se le encargó el informe por el consejo de Indias, justo antes de que llegaran a España Juvara y luego Sachetti para construir el palacio real

32 A.G.I., Guadalajara 420, fols. 266 y 267.

33 A.G.I., Guadalajara 420, fols. $268-269$ vto.

34 A.G.I., Guadalajara 420, fols. 269-270.

35 A.G.I., Guadalajara 420, fols. 270 y 270 vto. No hemos encontrado en el Archivo General de Indias la documentación complementaria a la analizada que Albuerne dijo que pensaba mandar a Madrid. 
Nuevo de Madrid, Ribera era el principal arquitecto de la capital y del reino. Su labor más significativa era la de maestro mayor de la villa de Madrid, cargo en el que había sucedido a Teodoro Ardemans, el cual había publicado en 1724 un tratado sobre la materia que se debatía: Fluencias de la tierra y curso subterráneo de las aguas. Ribera, por su parte, realizó numerosos de estos "viajes de agua" o conducciones subterráneas, así como fuentes y también intervino en el alcantarillado de Madrid, por lo que dominaba lo que proponía Buzeta en su proyecto ${ }^{36}$. Su importancia y experiencia sería la causa por la que el consejo de Indias lo elegiría para informar sobre la empresa novohispana.

Ribera no sólo tenía una gran formación práctica, también contaba con una sólida formación teórica y una nutrida biblioteca, de la que conocemos su inventario, con 124 volúmenes. Formada por los tratados de Vitruvio, Serlio, Palladio, Scamozzi, Montano, Cataneo, de Vries, Dietterlin o du Cerceau, contó lógicamente también con obras españolas, como las de Palomino, Carbonell, Torija o el referido Ardemans. Tuvo importantes obras de matemáticas, ingeniería y fortificación militar, pero cabe destacar que poseyera dos obras muy vinculadas a las conducciones de agua, como la Aritmética inferior y geometría práctica y especulativa: origen de los nacimientos de las aguas dulces y gordas de esta coronada villa de Madrid, sus viajes subterráneos... de Juan Claudio Aznar de Polanco, publicada en 1727, y Dificultades vencidas y curso natural en que se dan reglas especulativas y prácticas para la limpieza y asseo de las calles de esta corte de José Alonso de Arce, de $1735^{37}$.

También sabemos que Ribera tuvo el libro que cita en el informe para Guadalajara, Della trasportatione dell'obellisco vaticano et della fabriche di nostro signore papa Sisto $V$ de Domenico Fontana ${ }^{38}$. Asimismo, debió de contar o consultar De re aedificatoria de Alberti, que cita en el informe, pero que no es identificable entre los que aparecen en su inventario, en el que hay algunos de los que se dan tan pocos datos que no se pueden reconocer. Igualmente, pudo tener o leer las Historias de Tácito que hacen referencia al episodio en el que Moisés encontró agua para el pueblo de Israel que estaba sediento y que recoge en su informe para Nueva España. Como en el caso anterior, tampoco en esta ocasión es reconocible en el inventario de su biblioteca.

Sin duda, cabe considerar que Ribera tenía un conocimiento actualizado sobre las construcciones de agua, particularmente por tener, en primer lugar, las obras de Ardemans, que había sido, entre otras cosas, fontanero mayor, veedor de las conducciones de agua y maestro mayor de las fuentes de Madrid ${ }^{39}$. Con Ardemans llegó a trabajar Ribera y luego fue su sucesor al frente de las obras de la villa de Madrid, por lo que es seguro que conocería todas sus obras, tanto teóricas como prácticas. De igual modo, también conocía Ribera a Aznar de Polanco, no sólo autor de la obra antes referida, también del Tratado de los quatro elementos, origen y nacimiento de las aguas y fuentes de Madrid y sus viajes subterráneos. Las obras de Ardemans, Aznar de Polaco y José Alonso de Arce eran las principales fuentes españolas sobre conducciones de agua en época de Ribera ${ }^{40}$.

Además, como ya vimos, Ribera había llevado a la práctica no pocos de estos viajes de agua, tanto para el ayuntamiento de Madrid, como para la corona, de igual modo que había reparado y ejecutado diversas fuentes en la capital del reino. Se da la circunstancia de que en Madrid el abastecimiento de agua había sido realizado tradicionalmente de forma subterránea, debido a la riqueza del subsuelo de la capital y de su entorno, lo cual tuvo trascendencia en su organización

\footnotetext{
36 Sobre Ribera son esenciales Verdú RuIz, 1988 y, sobre todo, Verdú RuIZ, 1998.

37 Verdú Ruiz, 1998: 57-86.

38 VERdú RuIZ, 1998: 80.

39 Sobre Ardemans, además de su tratado -Ardemans, 1724-, son esenciales los estudios de Blasco EsQuivias, 1991; 1992 y 1998.

40 Véase Guerra Chavarino, 2006: 419 y ss.
}

Arch. esp. arte, LXXXVII, 348, OCTUBRE-DICIEMBRE 2014, 351-364 ISSN: 0004-0428, eISSN: 1988-8511, doi: 10.3989/aearte.2014.22 
urbana $^{41}$. Con tal bagaje no puede extrañar que Ribera asumiese con naturalidad la conducción de agua subterránea que proponía Buzeta para la Guadalajara, asumiendo el axioma de Ardemans: "si el manantial está profundo, se necesitan minas, si está quasi superficial, zanja abierta" 42 .

No obstante, Ribera en su informe, más que demostrar un amplio conocimiento sobre la más moderna tratadística relativa a la conducción de agua, parece que quiso probar una superioridad moral. La larga digresión que hace sobre Fontana y el obelisco de San Pedro del Vaticano apunta en tal sentido, igual que la alusión a Moisés. Resulta significativo que tampoco hiciese referencia alguna a las obras por él realizadas, como sí hizo por ejemplo Ardemans en su tratado ${ }^{43}$. No obstante, muestra su experiencia práctica, por ejemplo, al insistir en la necesidad de esperar al menos dos años para saber si el flujo de agua del acueducto es suficiente o al desaconsejar que se plantasen árboles en los márgenes del mismo. La insistencia de Ribera en esperar a que el agua fluyera durante largo tiempo, que también plantean Ardemans y Aznar de Polanco en sus tratados, cabe relacionarla con que en Madrid esos años bajó el nivel de sus acuíferos ${ }^{44}$.

Resulta del máximo interés que el informe de Ribera sobre el abastecimiento de agua de Guadalajara fuera el único que hizo para fuera de España. Sabemos que se contó con él para la expedición de títulos de arquitectura, la tasación de diversas obras, el reconocimiento de varias empresas y, particularmente, que informó sobre el polémico proyecto de Vicente Acero para la catedral nueva de Cádiz y para el entibamiento de la torre de la catedral nueva de Salamanca ${ }^{45}$. A partir de ahora, sabemos que también se le consultó sobre la obra novohispana tratada. Quizá en el futuro aparezcan nuevos informes suyos sobre otras obras de los virreinatos americanos.

En cualquier caso, nuestro informe evidencia ciertos detalles dignos de mención. En primer lugar, el carácter pedagógico de Ribera, tanto por lo que se refiere a sus citas históricas como a las cuestiones técnicas que aborda, en todo momento de meridiana claridad. Más significativo nos resulta la lección de humanidad que ofrece al soberbio Buzeta. Ello podría indicar que, a pesar de valorarse a sí mismo, Ribera no era una persona soberbia. En tal sentido, nos parece reveladora su idea de que hay que escuchar a todos los profesionales. En el otro lado de la balanza, es de resaltar lo mal parado que sale Buzeta. En primer lugar, en el plano personal, ya que resulta de una altivez insufrible, a lo que se suma la cuestión técnica, ya que, por ejemplo, creer que la afluencia de agua sería constante resulta absolutamente ingenuo. También parece un error de principiante flaquear la conducción de agua por árboles, ya que es bien sabido que posiblemente sus raíces terminen obstaculizando su paso. No es de extrañar por todo lo anterior que del informe se desprenda un buen concepto de Ribera, al que se le otorga el tratamiento de don que pone en evidencia el reconocimiento oficial y que, por el contrario, la figura del franciscano resulte hasta cómica debido a su soberbia, a pesar de ser aprobado su proyecto, aunque con muy significativos reparos.

Para terminar, hay que recordar que la obra del acueducto de Guadalajara fue culminada con éxito por Buzeta, lo que parece indicar que el franciscano debió de seguir las indicaciones de Ribera. No obstante, este éxito se convirtió muy pronto en un fracaso. El terremoto que sufrió Guadalajara en 1750 rompió su cañería, con lo que se interrumpió el abastecimiento de agua a

41 Sobre el abastecimiento de agua a Madrid véanse, además de las obras de Blasco Esquivias citadas en la nota 39, Montero Vallejo, 1982 y 1989; Landa Goñi, 1986 y Lopezosa Aparicio, 2005.

42 Ardemans, 1724: 88 y 89.

43 ARdemans, 1724: 90.

44 Montero Vallejo, 1989: 11-14.

45 Volvemos a remitir al respecto con carácter general a Verdú RUIZ, 1988 y a Verdú RuIZ, 1998. En concreto sobre la importante polémica sobre la catedral de Cádiz, que aquí sólo citamos, remitimos al menos a RoDRíGUEZ, 1992; MARÍAS, 2007 y MARÍAS, 2008. 
la ciudad. De nuevo se sucedieron las solicitudes de la audiencia al rey de ayuda, así como las peticiones de informes y la emisión de cédulas que intentaron salvar la situación. Se planteó tanto llevar a cabo un nuevo acueducto como reparar el realizado por Buzeta, que es lo que se realizó a la postre, de manera que en 1761 volvió a correr el agua por las fuentes de la plaza Mayor y de Santo Domingo, así como en siete conventos y casas particulares. Con posterioridad, se produjeron períodos de falta de agua y propuestas para hacer un nuevo acueducto. Tras la independencia de México y a lo largo del siglo XIX la situación se mantuvo de la misma forma, como si de una verdadera maldición se tratase: escasez, proyectos, fracasos, falta de recursos y así vuelta a empezar. No fue hasta comienzos del siglo XX cuando un nuevo acueducto puso definitivamente fin a la obra realizada por Buzeta y valorada por Ribera ${ }^{46}$.

\section{BIBLIOGRAFÍA}

Alberti, Leon Battista, Los diez libros de architectura, traducción de Francisco Lozano, Madrid, Alonso Gómez, 1582.

Angulo Íñiguez, Diego, Planos de monumentos arquitectónicos de América y Filipinas existentes en el Archivo de Indias. Estudio de los planos y de su documentación, 2 vols., Sevilla, Laboratorio de Arte de la Universidad de Sevilla, 1939.

Ardemans, Teodoro, Fluencias de la tierra y curso subterráneo de las aguas... Madrid, Francisco del Hierro, 1724.

Blasco Esquivias, Beatriz, Teodoro Ardemans y su entorno en el cambio de siglo (1661-1726). Aspectos de la arquitectura y el urbanismo madrileños de Felipe II a Carlos III, 2 vols., Madrid, Universidad Complutense de Madrid, 1991.

Blasco Esquivias, Beatriz, El tratado de Teodoro Ardemans sobre ordenanzas urbanas de Madrid (1719), Madrid, Gerencia municipal de urbanismo, 1992.

Blasco Esquivias, Beatriz, ;Agua va! La higiene urbana en Madrid (1561-1761), Madrid, Caja Madrid, 1998.

Calderón Quijano, José Antonio (dir.), Cartografia histórica de Nueva Galicia, Guadalajara, Universidad de Guadalajara, 1984.

Guerra Chavarino, Emilio, "Los viajes de agua de Madrid", Anales del instituto de estudios madrileños, n. ${ }^{\circ} 46$, Madrid, Instituto de estudios madrileños, 2006, pp. 419-466.

Jalomo Aguirre, Francisco, Gobernar el territorio entre descentralización y metropolización: el Patronato y el SIAPA como formas de gestión (1952-2006) y escenarios prospectivos, Guadalajara, Universidad de Guadalajara, 2011.

Landa Goñi, Jacinta, El agua en la higiene del Madrid de los Austrias, Madrid, Canal de Isabel II, 1986.

Laorden Ramos, Carlos, Obra civil en Ultramar del Real Cuerpo de Ingenieros, 2 vols., Madrid, Ministerio de Defensa, 2008.

López Cotilla, Manuel (atribución), Historia de la introducción de agua en Guadalajara desde su fundación hasta la fecha en la cual se han refundido todas las noticias importantes que ecsisten en la secretaría del Ayuntamiento y otras que se han adquirido en lo particular, Guadalajara, Imprenta del gobierno, 1842.

López Cotilla, Manuel (atribución), Historia de la introducción de agua en Guadalajara desde su fundación hasta la fecha en la cual se han refundido todas las noticias importantes que ecsisten en la secretaría del Ayuntamiento y otras que se han adquirido en lo particular, Guadalajara, Secretaría del ayuntamiento, 2013.

Lopezosa Aparicio, Concepción, El Paseo del Prado de Madrid. Arquitectura y desarrollo urbano en los siglos XVII y XVIII, Madrid, Fundación de apoyo a la historia del arte hispánico, 2005.

\footnotetext{
46 Véanse López Cotilla, 1842: 8 y ss.; Romero de Terreros, 1949: 127-129; Jalomo Aguirre, 2022: 158 y ss. y LAORDEN RAMOS, 2008: 40.
}

Arch. esp. arte, LXXXVII, 348, OCTUBRE-DICIEMBRE 2014, 351-364

ISSN: 0004-0428, eISSN: 1988-8511, doi: 10.3989/aearte.2014.22 
Marías, Fernando, "La catedral de Cádiz de Vicente Acero: la provocación de la arquitectura "crespa"”, Anuario del departamento de historia y teoría del arte, n. ${ }^{\circ}$ 19, Madrid, Universidad Autónoma de Madrid, 2007, pp. 79-103.

Marías, Fernando, "La catedral de Cádiz de Vicente Acero: la provocación de los textos", Anuario del departamento de historia y teoría del arte, n. ${ }^{\circ}$ 20, Madrid, Universidad Autónoma de Madrid, 2007, pp. 53-82.

Montero Vallejo, Manuel, Sótanos y duendes de Mantua y las aguas de Madrid, Madrid, Editora Nacional, 1982.

Montero Vallejo, Manuel, Problemas en el abastecimiento de agua a Madrid durante el siglo XVIII, Madrid, Instituto de estudios madrileños, 1989.

Ramos, Roberto, "Fray Pedro Antonio Buzeta, O.F.M. Introductor del agua potable en Guadalajara", Divulgación histórica n. $^{\circ}$ 5, México, Alberto María Carreño, 1942, pp. 218-226.

Rodríguez, Delfín, "Tradición e innovación en la arquitectura de Vicente Acero", Anales de arquitectura, n. ${ }^{\circ}$ 4, Valladolid, Universidad de Valladolid, 1992, pp. 37-49.

Romero de Terreros, Manuel, Los acueductos de México en la historia y en el arte, México, Instituto de investigaciones estéticas de la Universidad nacional autónoma de México, 1949.

Romo, Joaquín, Guadalajara. Apuntes históricos, biográficos, estadísticos y descriptivos de la capital del estado de Jalisco, desde su fundación por el conquistador Guzmán hasta nuestros días, México, Irineo Paz, 1888.

Ruiz Gomar, Rogelio, "El acueducto de Guadalupe”, en Estudios acerca del arte novohispano. Homenaje a Elisa Vargas Lugo, México, Universidad Nacional Autónoma de México, 1983, pp. 121-145.

Tácito, Cayo Cornelio, Las historias, 3 vols., Madrid, Imprenta Real, 1744.

Varios autores, Obras hidráulicas prehispánicas y coloniales en América, 3 vols., Madrid, Castalia, 1994.

Velázquez-Gaztelu, Juan Pedro, Fundación de todas las iglesias, conventos y ermitas de Sanlúcar de Barrameda, edición de Manuel Romero Tallafigo, Sanlúcar de Barrameda, Asociación sanluqueña de encuentros con la historia y el arte, 1995.

Verdú Ruiz, Matilde, La obra municipal de Pedro de Ribera, Madrid, Ayuntamiento de Madrid, 1988.

Verdú Ruiz, Matilde, El arquitecto Pedro de Ribera (1681-1742), Madrid, Instituto de estudios madrileños, 1998.

Fecha de recepción: $10-\mathrm{X}-2013$

Fecha de aceptación: 28-I-2014 